\title{
ENFERMAGEM: TEORIA, CONCEITOS, PRINCIPIOS E PROCESSO
}

\author{
Wanda de Aguiar Horta *
}

HORTA, W.A. - Enfermagem: teoria, conceitos, princípios e processo. Rev. Esc. Enf. USP., 8(1) 7-15, 1974.

Desenvolve-se uma teoria que procura explicar a natureza da enfermagem, seu campo especifico e sua metodologia de trabalho. Fundamenta-se na teoria de Maslow para explicar ser a enfermagem um senvico prestado ao Homem visando assisti-lo no atendimento de suas necessidades básicas e desta maneira contribuir para mantê-lo em equilibrio no tempo e espaço, seja prevenindo desequilibrios, ou revertendo estes em equilibrio. Da teoria proposta inferem-se os conceitos de enfermagem, assistir, assistência e cuidados em enfermagem. Algumas proposiçōes e principios também são expostos. Tendo a Teoria das Necessidades Humanas Básicas por fundamento, estabelece-se a metodologia ou Processo de Enfermagem em 6 fases: histórico, diagnóstico, plano assistencial, plano de cuidados, evoluçāo e prognóstico. Salienta-se a importância do desenvolvimento de habilidades denominadas instrumentos básicos, para a execução do Processo de Enfermagem.

\section{INTRODUÇĀO}

A dicotomia de rumos com que se defronta a enfermagem desenvolver-se como ciência própria ou tornar-se a profissão de Assistentemédico - leva seus profissionais a se dividirem, indecisos, entre as duas correntes. Esta situação incômoca, de transição, é sentida em toda a literatura publicada nesta década.

- Livre docente da disciplina Fundamentos de Enfermagem. 
Acreditamos ser a enfermagem uma ciência aplicada, saindo hoje da fase empirica para a científica, desenvolvendo suas teorias, sistematizando seus conhecimentos, pesquisando e tornando-se dia a dia uma ciência independente.

Em vinte e cinco anos de vida profissional temos acumulado observaçð̋es, aprendido, estudado, refletido; enfim temos vivido a enfermagem. Isto nos levou a procurar desenvolver uma teoria que pudesse explicar a natureza da enfermagem, definir seu campo de ação específico, sua metodologia científica.

Nossa teoria de enfermagem foi desenvolvida a partir da Teoria de Maslow que se fundamenta nas necessidades humanas básicas. E o que vamos procurar expor a seguir:

\section{TEORIA DAS NECESSIDADES HUMANAS BÄSICAS}

\section{1 - A ENFERMAGEM E U⿴囗 SERVIÇO PRESTADO AO HOMEM}

- O Homem é parte integrante do Universo dinâmico e como tal sujeito a todas as leis que o regem, no tempo e no espaço.

- A dinâmica do Universo provoca mudanças que o levam a estados de equilibrio e dessequilíbrio no tempo e no espaço. Resulta, pois:

1. O Homem como parte integrante do Universo está sujeito a estados de equilibrio e desequilibrio no tempo e no espaço.

- O Homem se distingue dos demais seres do Universo por sua capacidade de reflexão, por ser dotado do poder de imaginação e simbolizzção e por poder unir presente, passado e futuro.

- Estas características do Homem permitem sua Unicidade, Autenticidade e Individualidade.

- 0 Homem por suas características é também agente de mudanças no Universo dinâmico, no tempo e no espaço, consequentemente: 
2. O Homem, como agente de mudanf̧a é também a causa de equilibrio e desequilibrio em seu próprio dinamismo.

- Os desequilibrios geram no Homem necessidades que se caracterizam por estados de tensão conscientes ou inconscientes que o levam a buscar satisfação de tais necessidades para manter seu equilibrịo dinâmico no tempo e no espaço.

- As necessidades não atendidas ou atendidas inadequadamente trazem desconforto, e se este se prolonga é causa de doença.

- Estar com saúde é estar em equilibrio dinâmico no tempo e espaço.

\section{II - A ENFERMAGEM E PARTE INTEgRANTE DA EQUIPE DE SAODE. Do que resulta:}

- Como parte integrante da equipe de saúde, a enfermagem mantém o equilíbrio dinâmico, previne desequilíbrios e reverte desequilíbrios em equilíbrio do Homem, no tempo e no espaço.

- 0 Homem tem necessidades básicas que precisam ser atendidas para seu completo bem-estar.

- O conhecimento do Homem a respeito do atendimento de suas necessidades $e$ limitado por seu próprio saber exigindo, por isto, 0 auxilio de profissional habilitado.

necessária.

- Em estados de desequilíbrio esta assistência se faz mais

- Todos os conhecimentos e técnicas acumuladas sobre a enfermagem dizem respeito ao cuidado do ser humano, isto $\hat{\epsilon}$, como atendêlo em suas necessidades básicas.

- A enfermagem assiste o Homem no atendimento de suas necessidades básicas, valendo-se para isto dos conhecimentose princípios científicos das ciências físico-químicas, biológicas e psicossociais. A conclusão será: 
III - A ENFERMAGEM COMO PARTE INTEGRANTE DA EQUIPE DE SAUDE IMPLEMENTA ESTADOS DE EQUILIBRIO, PREVINE ESTADOS DE DESEQUILIBBRIO E REVERTE DESEQUILIBBRIOS EM EQUILÍBRIO PELA ASSISTENCIA AO HOMEM NO ATENDIMENTO DE SUAS NECESSIDADES BÁSICAS, PROCURA SEMPRE RECONDUZIR O HOMEM A SITUAÇÃO DE EQUILÍBRIO DINÂMICO NO TEMPO E ESPAÇO.

Desta teoria decorrem conceitos, proposiçōes e princípios que fundamentam a ciência de enfermagem.

\section{CONCEITO, PROPOSIÇÕES E PRINCIIPIOS}

Partindo-se da teoria proposta o primeiro conceito que se impõe é o de enfermagem: - Enfermagem é a ciência e a arte de assistir o ser humano* (indivíduo, família e comunidade) no atendimento de suas necessidades básicas, de tomá-lo independente desta assistência, quando possível, pelo ensino do auto-cuidado; de recuperar, manter e promover a saúde em colaboração com outros profissionais.

Assistir em enfermagem é: fazer pelo ser humano tudo aquilo que ele não pode fazer por si mesmo; ajudar ou auxiliar quando parcialmente impossibilitado de se autocuidar; orientar ou ensinar, supervisionar e encaminhar a outros profissionais.

Destes conceitos algumas proposições podem ser inferidas:

- As funçōes da enfermeira podem ser consideradas em tres áreas ou campos de ação distintos: a) Ȧrea especifica - assistir o ser humano no atendimento de suas necessidades básicas e torná-lo independente desta assistência, quando possível, pelo ensino do auto-cuidado; b) Área de interdependência ou de colaboração - a sua atividade na equipe de saúde nos aspectos de manutenção, promoção e recuperação da saúde;c) Área social dentro de sua atuação como uma profissional a serviço da sociedade, função de pesquisa, ensino, administração, responsabilidade legal e de participação na associação de classe.

- A expressäo sar humano utilizada neste trabalho significará sempre - individuo, famtlia e comunidade. 
- A ciência da enférmagem compreende o estudo das necessidades humanas básicas, dos fatores que alteram sua manifestação e atendimento, e na assistência a ser prestada.

Alguns princípios podem também ser deduzidos:

- A enfermagem respeita e mantém a unicidade, autenticidade $\mathrm{e}$ individualidade do Homem. ou desequilrbrio.

- A enfermagem é prestado ao Homem e não à sua doença

- Todo o cuidado de enfermagem é preventivo, curativo e de reabilitação.

- A enfermagem reconhece o Homem como membro de uma famnlia e de uma comunidade.

- A enfermagem reconhece o ser humano como elemento participante ativo no seu auto-cuidado.

Para que a enfermagem atue eficientemente, necessita desenvolver sua metodologia de trabalho que está fundamentada no método científico. Este método de atuação da enfermagem é denominado processo de enfermagem.

\section{PROCESSO DE ENFERMAGEM}

É a dinâmica das açōes sistematizadas e interrelacionadas que visa a assistência ao ser humano.

0 processo de enfermagem caracteriza-se pelo interrelacionamento e dinamismo de suas fases ou passos.

Distinguem-se seis fases ou passos. A interrelação e a igual importância destas fases no processo podem ser representadas graficamente (anexo 1), por um hexágono, cujas faces são vetores bi-orientados, querendo se assim mostrar, também, a reiteração eventual de procedimentos. No centro 
deste hexágono situar-se-ia o indivíduo, a familia e a comunidade.

- O primeiro passo do processo de enfermagem é o Histórico de enfermagem: roteiro sistematizado para o levantamento de dados (significativos para a enfermeira) do ser humano e que tornam possivel a identificaf̧ão de seus problemas.

Estes dados, convenientemente analisados e avaliados, levam ao segundo passo: Diagnóstico de enfermagem - A identificação das necessidades do ser humano que precisam de atendimento e a determinação, pela enfermeira, do grau de dependencia deste atendimento em natureza e em extensão.

O diagnóstico analisado e avaliado levará ao terceiro passo:

Plano assistencial: A determinação global da assistência de enfermagem que o ser humano deve receber diante do diagnóstico estabelecido.

Este plano assistencial é sistematizado em termos do conceito de assistir em enfermagem, isto é, encaminhamentos, supervisão (observação e controle), orientação, ajuda e execução de cuidados (fazer). Determinado o plano assistencial passa-se ao quarto passo:

Plano de cuidados: Implementação do plano assistencial pelo roteiro diário que coordena a ação da equipe de enfermagem na execução dos cuidados adequados ao atendimento das necessidades básicas e especiffcas do ser humano.

O plano de cuidados é avaliado diariamente, fornecendo os dados necessários para o quinto passo ou fase:

Evoluçāo de enfermagem: Relato diário das mudanças sures. sivas que ocorrem no ser humano, enquanto estiver sob assistência profissional.

Pela evolução é possivel avaliar a resposta do ser humano à assistência de enfermagem implementada. 
O estudo analítico e avaliação dos passos anteriores completa o hexágono com a sexta fase:

Prognóstico de enfermagem: Estimativa da capacidade do ser humano em atender suas necessidades básicas alteradas após a implementa. ção do plano assistencial e à luz dos dados fornecidos pela evolução de enfermagem.

Dadas as características já citadas do processo de enfermagem é possivel corrigir erros em qualquer uma das fases e também a previsão sjmultânea de todas as fases, assim é que ao fazermos o diagnóstico e mesmo na própria coleta de dados já teremos uma idéia do prognóstico; somente por razōes didáticas e de sistematização estas fases são separadas.

0 processo de enfermagem introduziu termos como ussistència e cuidado de enfermagem. Há diferença entre esta terminologia? Para muitos profissionais sāo sinônimos. De maneira restritiva e em alguns casos os dois termos podem ser usados com o mesmo significado, porém, de modo geral consideramos significados distintos, assim:

Assistência de enfermagem: E a aplicação, pela enfermeira, do processo de enfermagem para prestar o conjunto de cuidados e medidas que visam atender as necessidades básicas do ser humano.

Cuidado de enfermagem: E a ação planejada, deliberativa ou automática da enfermeira, resultante de sua percepção, observação e análise do comportamento, situação ou condição do ser humano.

0 cuidado de enfermagem pode implicar em várias atividades, por exemplo, a higiene oral - verificar o material que o paciente possui; avaliar sua capacidade de auto-cuidado; observar condiçōes da cavidade bucal; explicar o cuidado ao paciente; ensinat, se necessário, a técnica adequada de escovação; encaminhar ac cdontólogo; lavai o rnaterial utilizado; anotar, etc..

Para concluir esta exposição resta-nos ainda conceituar e enumerar os instrumentos básicos indispensáveis à enfermeira para que aplique o processo de enfermagem ou, em última análise, para que possa dar a assistência de enfermagem em qualidade e quantidade que se espera de um profissio- 
nal universitário.

Instrumentos básicos: São as habilidades, conhecimentos e atitudes indispensáveis para a execução de uma atividade. $\mathrm{Na}$ enfermagem estes instrumentos podem ser enumerados; sua sequeência não significa hierarquia pois todos têm o mesmo valor: observação, comunicação, aplicação do método científico, aplicação de princípios científicos, destreza manual, planejamento, avaliação, criatividade, trabalho em equipe, utilização dos recursos da comunidade.

As pesquisas que se têm desenvolvido e publicado em nosso paŕs parecem confirmar nossa teoria. Resta-nos esperar que novos estudos, trabalhos, pesquisas, e investigações confirmem totalmente ou a invalidem.

HORTA, W.A. - Nursing: theory, concepts, principles and process. Rev. Esc. Enf. USP, 8(1): 5-15, 1974.

Development of a theory that pursuit to explain the nursing's nature, specific field and work methodology. It is founded on Maslow's theory to achieve that nursing is a service to Man with the object of assist him to get their basic needs and by this manner contribue to maintain him in equilibrium in time and space; to prevent the human lost of balance or, in such a case, to revert this lost in new states of equilibrium. From this theory results the concepts of nursing: to assist, assistance and care in nursing. Also some propositions and principles are proposed. The development of a methodology is based upon this Human Basic Needs Theory, related and consequently Nursing Process is based in six phases: Nursing History, Nursing Diagnosis, Assistencial plan, Nursing care plan, Evolution and Nursing Prognosis. It is emphasized the concerning of the development of skills named basic tools to be possible the execution of the Nursing Process.

\section{REFERENCIAS BIBLIOGRÁFICAS}

HORTA, W.A. - Conceito de enfermagem. Rev. Esc. Enf. USP. 2 (2): 1-5 set. 1968.

HORTA, W.A. - Nota preliminar sobre o histórico de enfermagem. Rev. 
Esc. Enf. USP, 3 (2): 33-38, set. 1969.

HORTA, W.A. - Contribuição a uma teoria de enfermagem. Rev. Bras de Enf., $22(3,4,5,6):$ 119-125, jul. - dez. 1970.

HORTA, W.A. - Dois instrumentos básicos em enfermagem. Rev. Esc. Enf. USP, 4 (1 e 2): 3, mar-set. 1970.

HORTA, W.A. - Metodologia do processo de enfermagem. Rev. Bras de Enf., 24 (6): 81, out.-dez. 1971.

HORTA, W.A. - Processo de enfermagem. Ciência e Cultura, 24 (6): 534, jun. 1972. Suplemento.

HORTA, W.A. - Teoria das necessidades humanas básicas. Ciência e Cultura, 25(6):568, jun. 1973. Suplemento.

ROGERS, M. - Educational rerolution in nursing. New York, MacMillan Co., 1961.

ROGERS, M. - The theoretical basics of nursing. Davis Co., Philadelphia, 1972. 


\section{ANEXO 1}

\section{PROCESSO DE ENFERMAGEM}

E a dinàmica das ações sistematizadas que visa a assistência profissional ao individuo. família e comunidade.

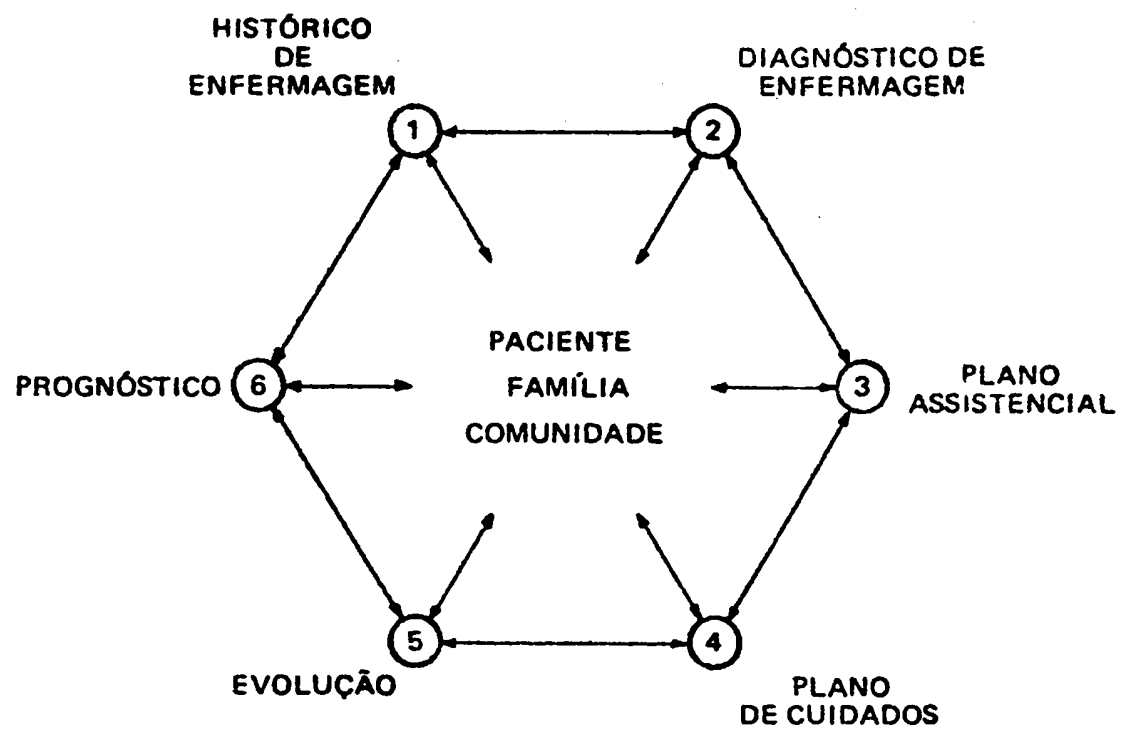




\section{EUNCÕES DA ENFERMEIRA}

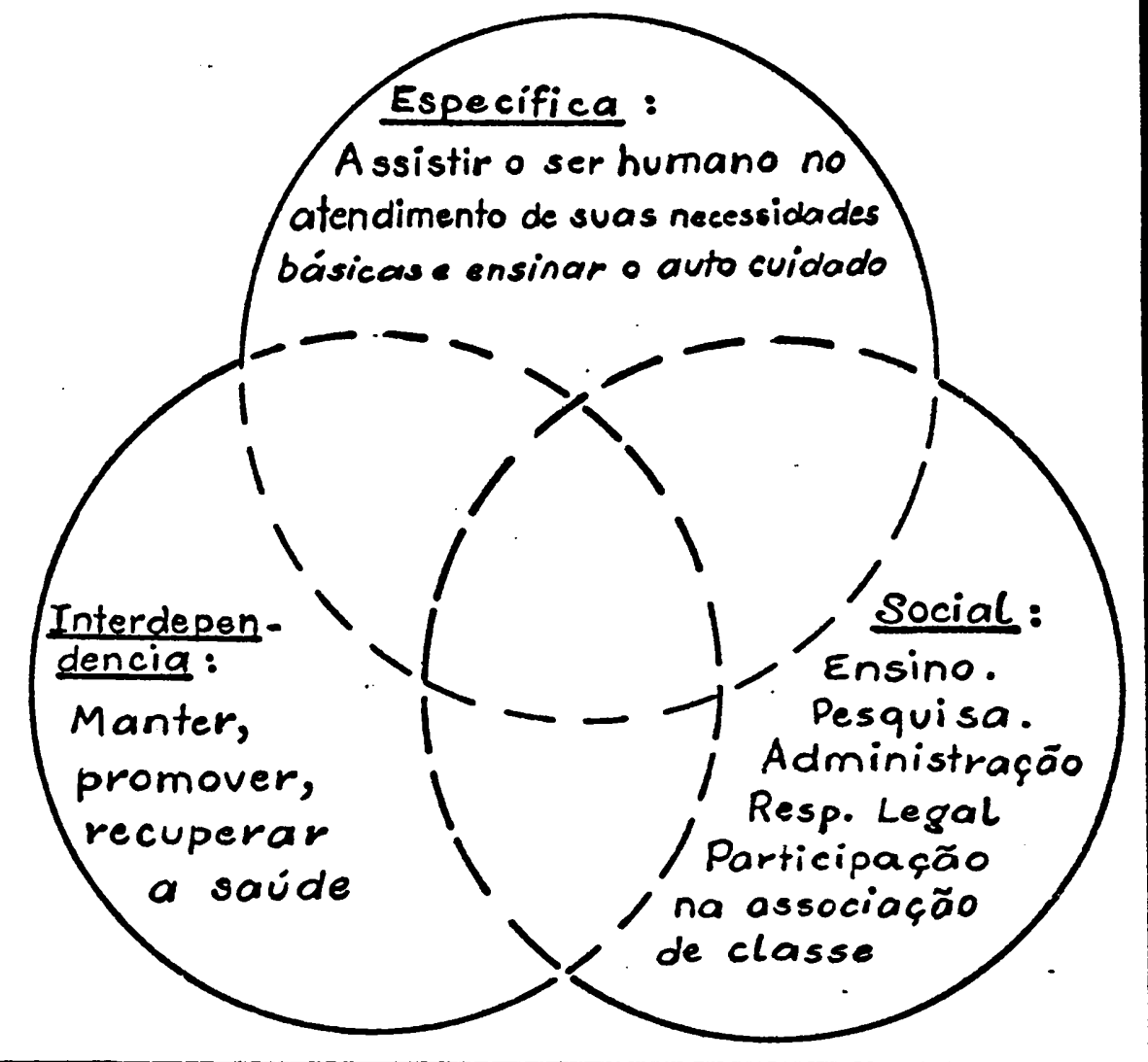

